\title{
Configuration Space Models for Spaces of Maps from a Riemann Surface to Complex Projective Space
}

\author{
By
}

Kohhei YamaguCHI*

\begin{abstract}
Let $\operatorname{Map}_{d}^{*}\left(\mathrm{M}_{g}, \mathbb{C P}^{n-1}\right)$ denote the space consisting of all basepoint preserving continuous maps of degree $d$ from a compact Riemann surface $\mathrm{M}_{g}$ of genus $g$ into a $(n-1)$-dimensional complex projective space $\mathbb{C P}^{n-1}$. In this paper, we construct a finite dimensional configuration space model $\mathrm{SP}_{n}^{d}\left(\mathrm{M}_{g}^{\prime}\right)$ for the infinite dimensional space $\operatorname{Map}_{d}^{*}\left(\mathrm{M}_{g}, \mathbb{C P}^{n-1}\right)$ and show that the Atiyah-Jones type theorem (cf. [1], [12]) holds for this model.
\end{abstract}

\section{$\S 1 . \quad$ Introduction}

Let $\mathrm{M}_{g}$ be a closed Riemann surface of genus $g$. If $X \subseteq \mathbb{C P}^{m}$ is a complex projective variety, we denote by $\left[\mathrm{M}_{g}, X\right]$ the set consisting of all basepoint preserving homotopy classes of basepoint preserving continuous maps $f: \mathrm{M}_{g} \rightarrow X$. For a class $D \in\left[\mathrm{M}_{g}, X\right]$, we denote by $\operatorname{Map}_{D}^{*}\left(\mathrm{M}_{g}, X\right)$ the space consisting of all basepoint preserving continuous maps $f: \mathrm{M}_{g} \rightarrow X$ with $f \in D$. The corresponding space of holomorphic maps is denoted by $\operatorname{Hol}_{D}^{*}\left(\mathrm{M}_{g}, X\right)$. In this paper, the author would like to study a finite dimensional model of $\operatorname{Map}_{d}^{*}\left(\mathrm{M}_{g}, \mathbb{C P}^{n-1}\right)$ for a non-negative integer $d \in \mathbb{Z} \cong\left[\mathrm{M}_{g}, \mathbb{C P}^{n-1}\right]$.

Let $\mathrm{SP}^{d}(X)$ be the $d$-th symmetric product of a space $X$. By definition, this is the quotient space $X^{d} / \Sigma_{d}$, where the symmetric group $\Sigma_{d}$ of $d$

Communicated by K. Saito, Received February 25, 2002.

2000 Mathematics Subject Classification(s): Primary 55P10; Secondary 55P35, 55P15

Key words: Mapping space, homotopy type, labelled configuration space.

The author was supported in part by a grant from the Ministry of Education of Japan.

* Department of Information Mathematics, University of Electro-Communications, Chofugaoka, Chofu, Tokyo 182-8585, Japan.

e-mail: kohhei@im.uec.ac.jp 
letters acts on $X^{d}$ in a natural way. An element of $\mathrm{SP}^{d}(X)$ may be identified with a formal linear combination $\alpha=\sum_{i=1}^{k} d_{i} x_{i}$, where $x_{1}, \ldots, x_{k}$ are distinct points of $X$ and $d_{1}, \ldots, d_{k}$ are positive integers such that $\sum_{i=1}^{k} d_{i}=d$. We shall refer to $\alpha$ as a "configuration" of points, the point $x_{i}$ having multiplicity $d_{i}$.

For an integer $n \geq 2$, let $\mathrm{SP}_{n}^{d}(X)$ denote the subspace of $\mathrm{SP}^{d}(X)$ given by

$$
\operatorname{SP}_{n}^{d}(X)=\left\{\sum_{i=1}^{k} d_{i} x_{i} \in \operatorname{SP}^{d}(X) \mid d_{i}<n \text { for all } i\right\} \subseteq \operatorname{SP}^{d}(X) .
$$

There is a filtration

$$
C_{d}(X)=\operatorname{SP}_{2}^{d}(X) \subseteq \mathrm{SP}_{3}^{d}(X) \subseteq \cdots \subseteq \operatorname{SP}_{d}^{d}(X) \subseteq \operatorname{SP}_{d+1}^{d}(X)=\operatorname{SP}^{d}(X),
$$

where $\mathrm{SP}_{2}^{d}(X)=C_{d}(X)$ is the unordered configuration space of $d$ distinct points in $X$. We shall consider the space $\mathrm{SP}_{n}^{d}\left(\mathrm{M}_{g}^{\prime}\right)$, where $x_{0} \in \mathrm{M}_{g}$ is a fixed basepoint and $\mathrm{M}_{g}^{\prime}$ denotes the space $\mathrm{M}_{g}^{\prime}=\mathrm{M}_{g}-\left\{x_{0}\right\}$.

When $g=0$, we can identify $\mathrm{M}_{0}=S^{2}=\mathbb{C} \cup\{\infty\}, \mathrm{M}_{0}^{\prime}=\mathbb{C}$ and $\operatorname{Map}_{d}^{*}\left(\mathrm{M}_{0}, \mathbb{C P}^{n-1}\right)=\Omega_{d}^{2} \mathbb{C P} P^{n-1} \simeq \Omega^{2} S^{2 n-1}$. Let $E_{d}: C_{d}(\mathbb{C}) \rightarrow \Omega_{d}^{2} \mathbb{C P}^{1}=\Omega_{d} S^{2}$ denote the electric field map defined by

$$
E_{d}\left(\sum_{j=1}^{d} z_{j}\right)(z)=\left\{\begin{array}{lll}
\sum_{j=1}^{d} 1 /\left(z-z_{j}\right) & \text { if } & z \in \mathbb{C} \\
0 & \text { if } & z=\infty
\end{array} \quad \text { for } z \in S^{2}=\mathbb{C} \cup \infty\right.
$$

Now we recall the following:

Theorem 1.1 ([2], [7], [8], [11], [12]).

(1) $E_{d}: C_{d}(\mathbb{C}) \rightarrow \Omega_{d}^{2} S^{2}$ is a homology equivalence up to dimension $[d / 2]$, where $[x]$ denotes the largest integer $\leq x$.

(2) If $n \geq 2$, the inclusion $i_{d}: \operatorname{Hol}_{d}^{*}\left(S^{2}, \mathbb{C P}^{n-1}\right) \rightarrow \Omega_{d}^{2} \mathbb{C P}^{n-1}$ is a homotopy equivalence up to dimension $(2 n-3) d$.

(3) If $n=2$, there is a stable homotopy equivalence $C_{d}(\mathbb{C}) \simeq_{s} \operatorname{Hol}_{[d / 2]}^{*}\left(S^{2}, S^{2}\right)$.

(4) If $n \geq 3$, there is a homotopy equivalence $\operatorname{SP}_{n}^{d}(\mathbb{C}) \simeq \operatorname{Hol}_{[d / n]}^{*}\left(S^{2}, \mathbb{C P}^{n-1}\right)$.

Remark. We say that a map $f: X \rightarrow Y$ is a homotopy equivalence (or homology equivalence) up to dimension $N$ if the induced homomorphism $f_{*}$ : $\pi_{j}(X) \rightarrow \pi_{j}(Y)\left(\right.$ or $\left.f_{*}: H_{j}(X, \mathbb{Z}) \rightarrow H_{j}(Y, \mathbb{Z})\right)$ is bijective when $j<N$ and is surjective when $j=N$. 
If $g=0, \mathrm{M}_{g}=S^{2}$ and this case was extensively well studied as above. So from now on, we consider the case $g \geq 1$. In this case, Segal already gave the following finite dimensional model for $\operatorname{Map}^{*}\left(\mathrm{M}_{g}, \mathbb{C P}^{n-1}\right)$.

Theorem $1.2([12])$. If $g \geq 1$ and $n \geq 2$, then the inclusion map $i_{d}: \operatorname{Hol}_{d}^{*}\left(\mathrm{M}_{g}, \mathbb{C P}^{n-1}\right) \rightarrow \operatorname{Map}_{d}^{*}\left(\mathrm{M}_{g}, \mathbb{C P}^{n-1}\right)$ is a homology equivalence up to dimension $(d-2 g)(2 n-3)$.

The purpose of this paper is to study another finite dimensional model and the main result is as follows.

Theorem 1.3. Let $g \geq 1$ and $n \geq 2$. Then there is a map

$$
\mathrm{SP}_{n}^{d}\left(\mathrm{M}_{g}^{\prime}\right) \rightarrow \operatorname{Map}_{d}^{*}\left(\mathrm{M}_{g}, \mathbb{C P}^{n-1}\right)
$$

which is a homology equivalence up to dimension $D(d, n)$, where we take the number $D(d, n)$ to be

$$
D(d, n)=\left\{\begin{array}{lll}
{[d / 2]} & \text { if } & n=2, \\
{[d / n]-n+3} & \text { if } & n \geq 3 .
\end{array}\right.
$$

The idea of the proof of the above theorem is to prove the stabilized result (Theorem 2.1) using the scanning map and is to show that the unstability theorem (Theorem 3.1) holds using Arnold-Segal type filtration of $\mathrm{SP}^{d}\left(\mathrm{M}_{g}^{\prime}\right)$.

We conclude with some comments on the significance of the main result of this paper. First, since $\lim _{d \rightarrow \infty} D(d, n)=\infty$, we may regard the space $\mathrm{SP}_{n}^{d}\left(\mathrm{M}_{g}^{\prime}\right)$ as a finite dimensional model for the infinite dimensional space $\operatorname{Map}_{0}^{*}\left(\mathrm{M}_{g}, \mathbb{C P}^{n-1}\right)$. In principle, the result of this type may be regarded as one of Atiyah-Jones type theorems (cf. [1]), and the first outstanding result of this kind was proved by Segal in [12], which indeed provided the motivation of this paper. The problem of finding such models is also considered in several papers (e.g. [3], [6], [7], [9], [12]) and it is originally appeared in the areas of geometry and mathematical physics. Second, the problem of finding a Morse theoretic interpretation of Segal's theorem also arises in our case. So there may be reason to believe that theorems of this type might hold for wide situations, although it is known that there are many counter examples and we do not use Morse theoretical method for the proof.

\section{§2. Configuration Spaces and Scanning Maps}

In this section we shall recall the relative configuration spaces and scanning maps. 
Relative configuration spaces. If $X$ be a connected space and $A$ is a closed subspace of $X$, we define

$\mathrm{SP}_{n}^{d}(X, A)=\left\{\sum_{i=1}^{k} d_{i} x_{i} \in \mathrm{SP}_{n}^{d}(X) \mid d_{i}<n\right.$ if $x_{i} \in X-A, x_{i} \neq x_{j}$ if $\left.i \neq j\right\} / \sim$,

where $\alpha \sim \beta$ if and only if $\alpha \cap(X-A)=\beta \cap(X-A)$. Thus, for $\operatorname{SP}_{n}^{d}(X, A)$, points in $A$ are "ignored". We also remark that $\operatorname{SP}_{n}^{d}(X, \emptyset)=\operatorname{SP}_{n}^{d}(X)$ if $A=\emptyset$. If $A \neq \emptyset$, there is a natural inclusion map $\operatorname{SP}_{n}^{d}(X, A) \stackrel{\subseteq}{\rightarrow} \operatorname{SP}_{n}^{d+1}(X, A)$ given by "adding a fixed point in $A$ ". We define $\operatorname{SP}_{n}(X, A)=\bigcup_{d \geq 1} \operatorname{SP}_{n}^{d}(X, A)$.

Stabilization maps. There is a stabilization map $s_{d}: \operatorname{SP}_{n}^{d}\left(\mathrm{M}_{g}^{\prime}\right) \rightarrow$ $\mathrm{SP}_{n}^{d+1}\left(\mathrm{M}_{g}^{\prime}\right)$ which is defined as follows. Let $\left\{U_{d}: d \geq 1\right\}$ be a closed neighborhoods of the basepoint $x_{0} \in \mathrm{M}_{g}$ such that, $U_{d} \supsetneqq U_{d+1}$ for each $d$ and each $U_{d}$ is homeomorphic to a closed 2-disk. Let us choose $z_{d} \in U_{d}-U_{d+1}$ for each $d$. Then define the stabilization map $s_{d}: \mathrm{SP}_{n}^{d}\left(\mathrm{M}_{g}^{\prime}\right) \rightarrow \mathrm{SP}_{n}^{d+1}\left(\mathrm{M}_{g}^{\prime}\right)$ by

$$
\begin{gathered}
\mathrm{SP}_{n}^{d}\left(\mathrm{M}_{g}^{\prime}\right) \simeq \mathrm{SP}_{n}^{d}\left(\mathrm{M}_{g}-U_{d}\right) \longrightarrow \mathrm{SP}_{n}^{d+1}\left(\mathrm{M}_{g}-U_{d+1}\right) \simeq \mathrm{SP}^{d+1}\left(\mathrm{M}_{g}^{\prime}\right) \\
\sum_{i=1}^{k} d_{i} x_{i} \longrightarrow z_{d}+\sum_{i=1}^{k} d_{i} x_{i}
\end{gathered}
$$

Scanning maps. To investigate the space $\lim _{d \rightarrow \infty} \mathrm{SP}_{n}^{d}\left(\mathrm{M}_{g}^{\prime}\right)$, we use the "scanning map", which we shall explain as follows. First, because $\mathrm{M}_{g}^{\prime}$ is parallelizable, if we choose a sufficiently small $\epsilon>0$, for each $z \in \mathrm{M}_{g}^{\prime}$ we can choose a canonical open set $U(z)$ such that it is homeomorphic to $U$ and it moves continuously when $z$ moves continuously.

Let $\alpha=\sum_{j=1}^{k} d_{j} x_{j} \in \mathrm{SP}_{n}^{d}\left(\mathrm{M}_{g}^{\prime}\right)$ and consider the map $s_{n}^{d}(\alpha): \mathrm{M}_{g}^{\prime} \rightarrow$ $\mathrm{SP}_{n}(\bar{U}, \partial \bar{U})$ given by $z \mapsto U(z) \cap \alpha \in \mathrm{SP}_{n}(\bar{U}(z), \partial \bar{U}(z)) \cong \mathrm{SP}_{n}(\bar{U}, \partial \bar{U})$.

Since $U(z) \cap \alpha$ is an empty configuration if $z \rightarrow x_{0}$ in $\mathrm{M}_{g}$, the map canonically extends to the map $s_{n}^{d}(\alpha): \mathrm{M}_{g} \rightarrow \mathrm{SP}_{n}(\bar{U}, \partial \bar{U})$. Note that $s_{n}^{d}(\alpha)$ is a basepoint-preserving map: the point $x_{0}$ is always mapped to the empty con-

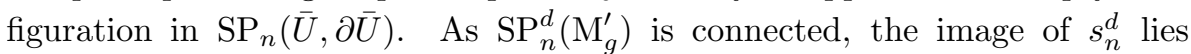
in a connected component of $\operatorname{Map}^{*}\left(\mathrm{M}_{g}, \mathrm{SP}_{n}(\bar{U}, \partial \bar{U})\right)$, which we denote by $\operatorname{Map}_{d}^{*}\left(\mathrm{M}_{g}, \operatorname{SP}_{n}(\bar{U}, \partial \bar{U})\right)$. So we have $s_{n}^{d}: \mathrm{SP}_{n}^{d}\left(\mathrm{M}_{g}^{\prime}\right) \rightarrow \operatorname{Map}_{d}^{*}\left(\mathrm{M}_{g}, \operatorname{SP}_{n}^{d}(\bar{U}, \partial \bar{U})\right)$. However, because there is a homotopy equivalence $\operatorname{SP}_{n}(\bar{U}, \partial \bar{U}) \simeq \mathbb{C P}^{n-1}([7])$, we obtain the map $s_{n}^{d}: \mathrm{SP}_{n}^{d}\left(\mathrm{M}_{g}^{\prime}\right) \rightarrow \operatorname{Map}_{d}^{*}\left(\mathrm{M}_{g}, \mathbb{C P}^{n-1}\right)$.

If we consider the homotopy commutative diagram

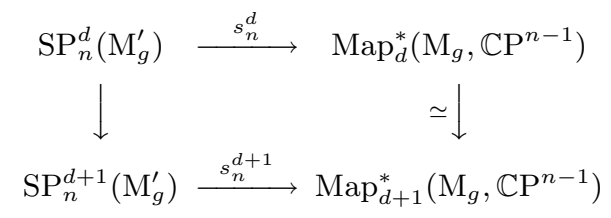


we have the stabilized scanning map $S: \lim _{d \rightarrow \infty} \mathrm{SP}_{n}^{d}\left(\mathrm{M}_{g}^{\prime}\right) \rightarrow \mathrm{Map}_{0}^{*}$ $\left(\mathrm{M}_{g}, \mathbb{C P}^{n-1}\right)$.

Theorem 2.1. $\quad S: \lim _{d \rightarrow \infty} \mathrm{SP}_{n}^{d}\left(\mathrm{M}_{g}^{\prime}\right) \stackrel{\simeq}{\rightarrow} \operatorname{Map}_{0}^{*}\left(\mathrm{M}_{g}, \mathbb{C P}^{n-1}\right)$ is a homotopy equivalence when $n \geq 3$ and a homology equivalence when $n=2$.

Proof. The proof repeats the proof of Proposition 4.2 in [12] almost wordfor-word. Alternatively, S. Kallel [10] also obtained this result.

\section{§3. Unstabilized Result}

In this section, we prove Theorem 1.3. From now on, we assume $g \geq 1$.

Lemma 3.1. $\quad s_{d}: \mathrm{SP}^{d}\left(\mathrm{M}_{g}^{\prime}\right) \rightarrow \mathrm{SP}^{d+1}\left(\mathrm{M}_{g}^{\prime}\right)$ is a homology equivalence up to dimension $d$.

Proof. The proof is given using the method of Proposition (A.2) of [12], or that of $(2.4)$ of $[6]$.

We remark that there is a homotopy equivalence $\operatorname{Map}_{d}^{*}\left(\mathrm{M}_{g}, \mathbb{C P}^{n-1}\right) \simeq$ $\operatorname{Map}_{0}^{*}\left(\mathrm{M}_{g}, \mathbb{C P}^{n-1}\right)$ for any $d \neq 0$, and let us consider the composite of maps

$$
\mathrm{SP}_{n}^{d}\left(\mathrm{M}_{g}^{\prime}\right) \stackrel{\text { natural map }}{\longrightarrow} \lim _{d^{\prime} \rightarrow \infty} \mathrm{SP}_{n}^{d^{\prime}}\left(\mathrm{M}_{g}^{\prime}\right) \stackrel{S}{\simeq} \operatorname{Map}_{0}^{*}\left(\mathrm{M}_{g}, \mathbb{C P}^{n-1}\right)
$$

Then the main result (Theorem 1.3) easily follows from Theorem 2.1 and the following unstabilized result.

Theorem 3.1. $\quad s_{d}: \mathrm{SP}_{n}^{d}\left(\mathrm{M}_{g}^{\prime}\right) \rightarrow \mathrm{SP}_{n}^{d+1}\left(\mathrm{M}_{g}^{\prime}\right)$ is a homology equivalence up to dimension $D(d, n)$, where (as in Theorem 1.3)

$$
D(d, n)=\left\{\begin{array}{lll}
{[d / 2]} & \text { if } & n=2 \\
{[d / n]-n+3} & \text { if } & n \geq 3 .
\end{array}\right.
$$

Proof. From now on, we write $\mathrm{SP}_{n}^{d}=\mathrm{SP}_{n}^{d}\left(\mathrm{M}_{g}^{\prime}\right)$ and $\mathrm{SP}^{d}=\mathrm{SP}^{d}\left(\mathrm{M}_{g}^{\prime}\right)$. If $n=2$, since $\mathrm{SP}_{2}^{d}=C_{d}\left(\mathrm{M}_{g}^{\prime}\right)$, the assertion follows from the appendix of [12]. So we assume $n \geq 3$. The proof is by induction on $d$.

If $d<n, \mathrm{SP}_{n}^{d}=\mathrm{SP}^{d}$ and using Lemma 3.1, the assertion clearly holds. So we assume that there exists some number $d \geq n$ such that, for any $k<d$ the induced homomorphism $\left(s_{k}\right)_{*}: H_{j}\left(\mathrm{SP}_{n}^{k}, \mathbb{Z}\right) \rightarrow H_{j}\left(\mathrm{SP}_{n}^{k+1}, \mathbb{Z}\right)$ is bijective when $j<D(k, n)=[k / n]-n+3$ and is surjective when $j=D(k, n)$. 
In this situation, we shall prove that $\left(s_{d}\right)_{*}: H_{j}\left(\mathrm{SP}_{n}^{d}, \mathbb{Z}\right) \rightarrow H_{j}\left(\mathrm{SP}_{n}^{d+1}, \mathbb{Z}\right)$ is bijective when $j<D(d, n)$ and surjective when $j=D(d, n)$.

First, we note that the map $s_{d}$ naturally extends to an open embedding $\mathrm{SP}_{n}^{d} \times \mathbb{C} \rightarrow \mathrm{SP}_{n}^{d+1}$ and this induces the map $\bar{s}_{d}: \overline{\mathrm{SP}_{n}^{d+2}} \rightarrow \overline{\mathrm{SP}_{n}^{d} \times \mathbb{C}}=\mathrm{SP}_{n}^{d} \wedge S^{2}$, where $\bar{X}$ denotes the one-point compactification of a locally compact space $X$. Then we define the homomorphism $\left(s_{d}\right)_{c}^{*}: H_{c}^{k}\left(\mathrm{SP}_{n}^{d}, \mathbb{Z}\right) \rightarrow H_{c}^{k+2}\left(\mathrm{SP}_{n}^{d+1}, \mathbb{Z}\right)$ by the composite of homomorphisms

$$
H_{c}^{k}\left(\mathrm{SP}_{n}^{d}, \mathbb{Z}\right) \stackrel{\text { suspension iso. }}{\cong} H_{c}^{k+2}\left(\mathrm{SP}_{n}^{d} \times \mathbb{C}, \mathbb{Z}\right) \stackrel{\bar{s}_{d}^{*}}{\longrightarrow} H_{c}^{k+2}\left(\mathrm{SP}_{n}^{d+2}, \mathbb{Z}\right) .
$$

We also remark that $\mathrm{SP}^{d}$ is an open $d$-dimensional complex manifold. Since $\mathrm{SP}_{n}^{d} \subset \mathrm{SP}^{d}$ is an open subset, $\mathrm{SP}_{n}^{d}$ is also an open complex manifold of the same dimension and there is a commutative diagram

$$
\begin{array}{ccc}
H_{j}\left(\mathrm{SP}_{n}^{d}, \mathbb{Z}\right) \stackrel{\left(s_{d}\right)_{*}}{\longrightarrow} & H_{j}\left(\mathrm{SP}_{n}^{d+1}, \mathbb{Z}\right) \\
\cong \mid \text { P.d. } & \cong \mid \text { P.d. } \\
H_{c}^{2 d-j}\left(\mathrm{SP}_{n}^{d}\right) \stackrel{\left(s_{d}\right)_{c}^{*}}{\longrightarrow} H_{c}^{2 d+2-j}\left(\mathrm{SP}_{n}^{d+1}\right)
\end{array}
$$

where the vertical isomorphisms are Poincáre duality isomorphisms. Hence, it follows from the induction hypothesis that the following holds:

$(*)_{d}$ If $k<d$, the induced homomorphism $\left(s_{k}\right)_{c}^{*}: H_{c}^{j}\left(\mathrm{SP}_{n}^{k}\right) \rightarrow H_{c}^{j+2}\left(\mathrm{SP}_{n}^{k+1}\right)$ is bijective when $j>N(k, n)=2 d-[k / n]+n-3$ and is surjective when $j=N(k, n)$.

Under the above assumption $(*)_{d}$, it follows from the above commutative diagram that it suffices to prove that $\left(s_{d}\right)_{c}^{*}: H_{c}^{j}\left(\mathrm{SP}_{n}^{d}\right) \rightarrow H_{c}^{j+2}\left(\mathrm{SP}_{n}^{d+1}\right)$ is bijective when $j>N(d, n)=2 d-[d / n]+n-3$ and is surjective when $j=$ $N(d, n)$.

Let $P_{m}^{d} \subset \mathrm{SP}^{d}$ denote the subspace $P_{m}^{d}=\left\{\xi+n \cdot \eta \in \mathrm{SP}^{d}: \operatorname{deg}(\eta) \geq m\right\}$, where we take $\operatorname{deg}(\eta)=\sum_{j=1}^{k} d_{j}$ if $\eta=\sum_{j=1}^{k} d_{j} \cdot z_{j}$.

Then there is a Arnold-Segal type filtration

$$
\mathrm{SP}^{d}=P_{0}^{d} \supset P_{1}^{d} \supset \cdots \supset P_{[d / n]}^{d} \supset P_{[d / n]+1}^{d}=\emptyset,
$$

where $\mathrm{SP}_{n}^{d}=\mathrm{SP}^{d}-P_{1}^{d}$ and $P_{m}^{d}-P_{m+1}^{d} \cong \mathrm{SP}_{n}^{d-n m} \times \mathrm{SP}^{m}$ for $0 \leq m \leq[d / n]$. The stabilization map induces maps

$$
\left\{\begin{array}{l}
P_{m}^{d}-P_{m+1}^{d} \rightarrow P_{m}^{d+1}-P_{m+1}^{d+1} \\
P_{m}^{d} \rightarrow P_{m}^{d+1}
\end{array}\right.
$$


for each $0 \leq m \leq[d / n]$. Consider the commutative diagram

(i)

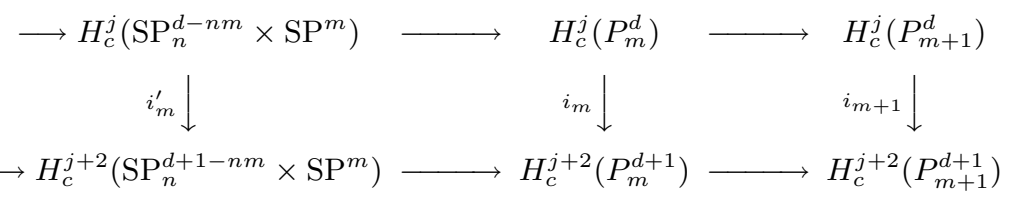

where the horizontal sequences are exact. We need the following result.

Lemma 3.2. Let $M(m)=2 d-(2 n-3) m-[d / n]-3+n$. If $1 \leq m \leq$ $[d / n]$, the induced homomorphism $i_{m}: H_{c}^{j}\left(P_{m}^{d}\right) \rightarrow H_{c}^{j+2}\left(P_{m}^{d+1}\right)$ is bijective when $j>M(m)$ and is surjective when $j=M(m)$.

Proof of Lemma 3.2. This is proved by downwards induction on $m$.

If $m=[d / n]$, since

$$
\left\{\begin{array}{l}
\operatorname{dim} P_{[d / n]}^{d}=2(d-n[d / n]+[d / n]) \leq N([d / n]) \\
\operatorname{dim} P_{[d / n]}^{d+1}=2(d-n[d / n]+[d / n]+1) \leq N([d / n])+2,
\end{array}\right.
$$

the assertion clearly holds. Assume that $i_{m+1}$ is bijective when $j>M(m+1)$ and is surjective when $j=M(m+1)$. Consider the commutative diagram

$$
\begin{array}{ccc}
H_{c}^{j}\left(\mathrm{SP}_{n}^{d-n m} \times \mathrm{SP}^{m}\right) & \stackrel{i_{m}^{\prime}}{\longrightarrow} \quad H_{c}^{j+2}\left(\mathrm{SP}_{n}^{d+1-n m} \times \mathrm{SP}^{m}\right) \\
\cong \downarrow \text { P.d. } & \cong \downarrow \mathrm{P} . \mathrm{d} . \\
H_{2 d-2 m(n-1)-j}\left(\mathrm{SP}_{n}^{d-n m} \times \mathrm{SP}^{m}\right) & \longrightarrow H_{2 d-2 m(n-1)-j}\left(\mathrm{SP}_{n}^{d+1-n m} \times \mathrm{SP}^{m}\right)
\end{array}
$$

Since $2 d-2 m(n-1)-j \leq D(d-n m) \Leftrightarrow j \geq M(m)$, it follows from the inductive hypothesis that $i_{m}^{\prime}$ is bijective when $j>M(m)$ and is surjective when $j=M(m)$. Hence using the Five Lemma, $M(m)>M(m+1)$ and the diagram (i), we can show that the assertion holds for the case $m$, too.

Consider the commutative diagram

(ii)

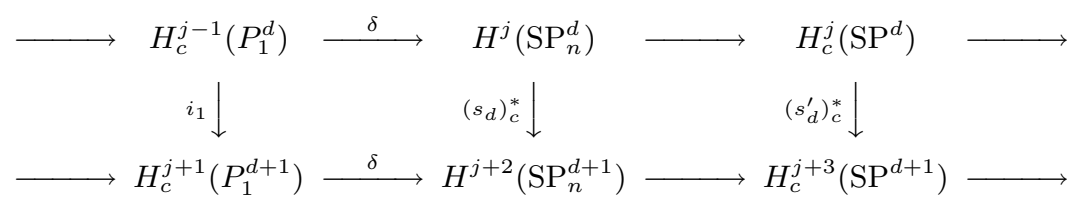

where the horizontal sequences are exact.

It follows from Lemma 3.1 and Poincáre duality that $\left(s_{d}^{\prime}\right)_{c}^{*}$ is bijective when $j>d$ and surjective when $j=d$. 
In general, we can write $d=n[d / n]+\epsilon$ for some $0 \leq \epsilon \leq n-1$. Then, because $(M(1)+1)-d=(n-1)[d / n]+\epsilon-(n-1) \geq 0, \max \{M(1)+1, d\}=$ $M(1)+1=2 d-n+1-[d / n]$. Hence it follows from Lemma 3.2 and (ii) that $\left(s_{d}\right)_{c}^{*}: H_{c}^{j}\left(\mathrm{SP}_{n}^{d}\right) \rightarrow H_{c}^{j+2}\left(\mathrm{SP}_{n}^{d+1}\right)$ is bijective when $j>M(1)+1$ and is surjective when $j=M(1)+1$.

However, since $N(d, n)=2 d-[d / n]+n-3 \geq M(1)+1$, the homomorphism $\left(s_{d}\right)_{c}^{*}: H_{c}^{j}\left(\mathrm{SP}_{n}^{d}\right) \rightarrow H_{c}^{j+2}\left(\mathrm{SP}_{n}^{d+1}\right)$ is bijective when $j>N(d, n)$ and is surjective when $j=N(d, n)$. This completes the proof of Theorem 3.1.

\section{$\S 4$. Homology Decompositions}

Finally, we remark some results concerning the homology of $\mathrm{SP}_{n}^{d}\left(\mathrm{M}_{g}^{\prime}\right)$.

We define the map $\tau^{d}: \mathrm{SP}_{n}^{d}\left(\mathrm{M}_{g}^{\prime}\right) \rightarrow \mathrm{SP}^{\infty}\left(\mathrm{SP}_{n}^{d-1}\left(\mathrm{M}_{g}^{\prime}\right)\right)$ by $\alpha=\sum_{j=1}^{d} z_{j} \mapsto$ $\sum_{j=1}^{d} \sum_{i \neq j} z_{i}$ for $\alpha \in \mathrm{SP}_{n}^{d}\left(\mathrm{M}_{g}^{\prime}\right)$, and this naturally extends to the homomorphism of monoids, $\tau^{d}: \mathrm{SP}^{\infty}\left(\mathrm{SP}_{n}^{d}\left(\mathrm{M}_{g}^{\prime}\right)\right) \rightarrow \mathrm{SP}^{\infty}\left(\mathrm{SP}_{n}^{d-1}\left(\mathrm{M}_{g}^{\prime}\right)\right)$.

Let $\mathrm{SP}_{n}^{d}\left(\mathrm{M}_{g}^{\prime}\right) / \mathrm{SP}_{n}^{d-1}\left(\mathrm{M}_{g}^{\prime}\right)$ denote the mapping cone of the map $s_{d-1}$ : $\mathrm{SP}_{n}^{d-1}\left(\mathrm{M}_{g}^{\prime}\right) \rightarrow \mathrm{SP}_{n}^{d}\left(\mathrm{M}_{g}^{\prime}\right)$, and for each $1 \leq m \leq d$, let $\tau_{m}^{d}: \mathrm{SP}^{\infty}\left(\mathrm{SP}_{n}^{d}\left(\mathrm{M}_{g}^{\prime}\right)\right) \rightarrow$ $\mathrm{SP}^{\infty}\left(\mathrm{SP}_{n}^{m}\left(\mathrm{M}_{g}^{\prime}\right) / \mathrm{SP}_{n}^{m-1}\left(\mathrm{M}_{g}^{\prime}\right)\right)$ be the composite of maps

$$
\begin{gathered}
\mathrm{SP}^{\infty}\left(\mathrm{SP}_{n}^{d}\left(\mathrm{M}_{g}^{\prime}\right)\right) \stackrel{\tau^{d}}{\rightarrow} \mathrm{SP}^{\infty}\left(\mathrm{SP}_{n}^{d-1}\left(\mathrm{M}_{g}^{\prime}\right)\right) \stackrel{\tau^{d-1}}{\rightarrow} \mathrm{SP}^{\infty}\left(\mathrm{SP}_{n}^{d-2}\left(\mathrm{M}_{g}^{\prime}\right)\right) \stackrel{\tau^{d-2}}{\rightarrow} \cdots \\
\ldots \stackrel{\tau^{m+1}}{\rightarrow} \mathrm{SP}^{\infty}\left(\mathrm{SP}_{n}^{m}\left(\mathrm{M}_{g}^{\prime}\right)\right) \rightarrow \mathrm{SP}^{\infty}\left(\mathrm{SP}_{n}^{m}\left(\mathrm{M}_{g}^{\prime}\right) / \mathrm{SP}_{n}^{m-1}\left(\mathrm{M}_{g}^{\prime}\right)\right)
\end{gathered}
$$

Proposition 4.1. The map

$$
\left(\tau_{1}^{d}, \ldots, \tau_{d}^{d}\right): \mathrm{SP}^{\infty}\left(\mathrm{SP}_{n}^{d}\left(\mathrm{M}_{g}^{\prime}\right)\right) \stackrel{\simeq}{\rightarrow} \prod_{m=1}^{d} \mathrm{SP}^{\infty}\left(\mathrm{SP}_{n}^{m}\left(\mathrm{M}_{g}^{\prime}\right) / \mathrm{SP}_{n}^{m-1}\left(\mathrm{M}_{g}^{\prime}\right)\right)
$$

is a homotopy equivalence.

Proof. The assertion easily follows from Lemma 2 of [4] and we omit the detail.

If we use the Dold-Thom Theorem [5], we also obtain the following:

Corollary 4.1. For a commutative ring $A$ and an integer $j \geq 1$,

$$
\oplus\left(\tau_{m}^{d}\right)_{*}: H_{j}\left(\mathrm{SP}_{n}^{d}\left(\mathrm{M}_{g}^{\prime}\right), A\right) \stackrel{\cong}{\rightrightarrows} \oplus_{m=1}^{d} H_{j}\left(\mathrm{SP}_{n}^{m}\left(\mathrm{M}_{g}^{\prime}\right) / \mathrm{SP}_{n}^{m-1}\left(\mathrm{M}_{g}^{\prime}\right), A\right)
$$

is an isomorphism. 


\section{Acknowledgements}

The author is indebted to M. A. Guest, A. Kozlowski and K. Shimakawa for numerous helpful conversations concerning the topology of labelled configuration spaces and related topics.

\section{References}

[1] Atiyah, M. F. and Jones, J. D. S., Topological aspects of Yang-Mills theory, Commun. Math. Phys., 59 (1978), 97-118.

[2] Cohen, F. R., Cohen, R. L., Mann, B. M. and Milgram, R. J., The topology of rational functions and divisors of surfaces, Acta Math., 166 (1991), 163-221.

[3] Cohen, R. L., Jones, J. D. S. and Segal, G. B., Floer's infinite dimensional Morse theory and homotopy theory, Progr. Math., 133 (1995), 297-325.

[4] Dold, A., Decompotion theorems for $S(n)$-complexes, Ann. of Math., 75 (1962), 8-16.

[5] Dold, A. and Thom, R., Quasifaserungen und unendliche symmetrische Produkte, Ann. of Math., 67 (1958), 239-281.

[6] Guest, M. A., Kozlowski, A. and Yamaguchi, K., The topology of spaces of coprime polynomials, Math. Z., 217 (1994), 435-446.

[7] Spaces of polynomials with roots of bounded multiplicity, Fund. Math., 116 (1999), 93-117.

[8] Stable splitting of the space of polynomials with roots of bounded multiplicity, J. Math. Kyoto Univ., 38 (1998), 351-366.

[9] Guest, M. A., The topology of the space of rational curves on a toric variety, Acta Math., 174 (1995), 119-145.

[10] Kallel, S., Configuration spaces and the topology of curves in projective space, Contemp. Math., 279 (2001), 151-175.

[11] Segal, G. B., Configuration spaces and iterated loop spaces, Invent. Math., 21 (1973), 213-221.

[12] $ـ$, The topology of spaces of rational functions, Acta Math., 143 (1979), 39-72. 